\title{
Q-learning vertical handover scheme in two-tier LTE-A networks
}

\author{
Ammar Bathich ${ }^{1}$, Mohd Asri Mansor ${ }^{2}$, Saiful Izwan Suliman ${ }^{3}$, Sinan Ghassan Abid Ali ${ }^{4}$ \\ ${ }^{1,2,3}$ Faculty of Electrical Engineering, Universiti Teknologi MARA, Malaysia \\ ${ }^{4}$ Faculty of Computer Technologies Engineering, Iraq University College, Iraq
}

\begin{tabular}{|c|c|}
\hline Article Info & ABSTRACT \\
\hline & \multirow{9}{*}{$\begin{array}{l}\text { Global mobile communication necessitates improved capacity and proper } \\
\text { quality assurance for services. To achieve these requirements, small cells } \\
\text { have been deployed intensively by long term evolution (LTE) networks } \\
\text { operators beside conventional base station structure to provide customers } \\
\text { with better service and capacity coverage. Accomplishment of seamless } \\
\text { handover between Macrocell layer (first tier) and Femtocell layer (second } \\
\text { tier) is one of the key challenges to attain the QoS requirements. Handover } \\
\text { related information gathering becomes very hard in high dense femtocell } \\
\text { networks, effective handover decision techniques are important to minimize } \\
\text { unnecessary handovers occurred and avoid Ping-Pong effect. In this work, } \\
\text { we proposed and implemented an efficient handover decision procedure } \\
\text { based on users' profiles using Q-learning technique in an LTE-A macrocell- } \\
\text { femtocell networks. New multi-criterion handover decision parameters are } \\
\text { proposed in typical/dense femtocells in microcells environment to estimate } \\
\text { the target cell for handover. The proposed handover algorithms are validated } \\
\text { using the LTE-Sim simulator under an urban environment. The simulation } \\
\text { results showed noteworthy reduction in the average number of handovers. }\end{array}$} \\
\hline Received Apr 1, 2020 & \\
\hline Revised Apr 11, 2020 & \\
\hline Accepted May 1, 2020 & \\
\hline Keywords: & \\
\hline LTE-A & \\
\hline Q-learning & \\
\hline Two-tier networks & \\
\hline Vertical handover & \\
\hline
\end{tabular}

Copyright @ 2020 Institute of Advanced Engineering and Science. All rights reserved.

Corresponding Author:

Ammar Bathich,

Faculty of Electrical Engineering, Universiti Teknologi MARA, 40450 Shah Alam, Selangor, Malaysia.

Email: ammarbat2003@gmail.com

\section{INTRODUCTION}

Dependency on using the handover procedure in long term evolution advanced (LTE-A) networks for the User Equipment UE mobility results in the reduction in wireless network complexity [1]. In spite of this advantage, the handover still experiences multifunction. For example, the handover decision is affected by carrier interferences and inflexibility [2-4]. This keeps us with the QoS requirements in high delay and loss during handover among cells [5]. The handover procedure will become more critical when the UE starts moving from the serving station to the target station [6]. Furthermore, the smooth handover technique needs to be periodic and fast, and the data transfer should not be lost and delayed. Even more, the increasing demands for using wireless broadband has led to the mobile network operators deploying more wireless cells of various types (macrocell (eNB) and femtocell (HeNB)) to fulfil the data traffic rate [7]. Thus, a handover between the two-tier networks will cause an unnecessary handover effect, handover failure and a drop in the performance of the wireless network. The Figure 1 shows eNB and HeNBs cells in E-UTRAN LTE-A architecture.

The standard handover decision in LTE-A is based on the received signal strength (RSS) and received signal quality (RSQ) parameters. Thus, the deployment of dense HeNBs inside the eNB cell would increase the interference. This interference reduces the RSQ by decreasing the RSS ratio in comparison to the Received Signal Strength Indicator RSSI. As a result, a degradation in handover performance is 
observed [8]. Moreover, the variation in transmitting power between the two types of wireless networks (eNB and HeNB) is another issue. When the HeNB location is near the eNB tower, the RSS of the HeNB will always be less than the eNB [9-12].

Many researches have worked on enhancing the handover procedure in the wireless networks in LTE-A. This has been achieved by reducing packet loss and packet delay and simultaneously increasing network throughput. LTE-A promises improvement in handover performance and guarantee of a satisfactory QoS for real time applications. However, there are still many concerns regarding the capability of adopting these schemes in real environments. This real environment deployment issue can be solved by minimizing the extra overhead of corresponding mechanisms and thus minimize calculation time.

The drawbacks that must be addressed in LTE-A handover technique could be summarized in three points: Firstly, current selection of handover decision parameters is based on RSS and RSQ parameters, which are inappropriate for high-density HeNB deployment in LTE-A networks. Secondly, long searching time/frequent handovers due to presence of multiple target cells which cause degradation of handover performance. Thirdly, inefficient in the standard handover scheme in the presence of HeNB cells yields to unnecessary handovers, handover failure and dropping the network performance.

Various works in the handover decision were proposed in [13-16] to find out the target station. In $[15,16]$, the mobility forecast of a user equipment is based on keep tracking of its previous three locations. Nevertheless, the researchers did not explain their methodologies for choosing the locations of UE as well as the distances among them, they did not take into consideration macrocell load. Furthermore, in [13, 14], the target station was selected based on the probability of the UE movement activity in systematic directions with fixed speed, nevertheless, this suggested method cannot be realized in real conditions. In this work, a novel handover decision structure is proposed for choosing the target station based on UE mobility history by applying a Q-learning based technique that takes a handover decision according to the current and past history of the environment. We compared our handover proposed algorithm with Suman work [17] in terms of average number of handovers. LTE-Sim simulator has been developed to evaluate the system performance. The remainder of the paper is structured as follows: Section 2 shows and discusses the Q-learning environment used. The proposed model has been discussed in section 3. Section 4 condenses the performance evaluation of the proposed work. Finally, section 5 summaries the paper.

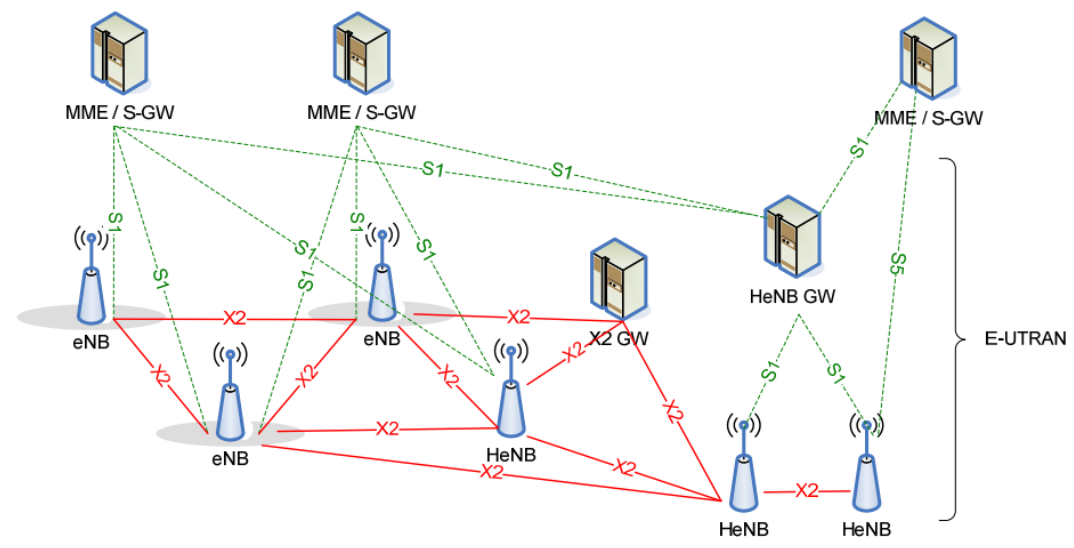

Figure 1. HeNBs in E-UTRAN architecture

\section{Q-LEARNING ALGORITHM}

Q-Learning is a type of machine learning technique where an agent attempts to find an ideal strategy from its history of movement inside a dynamic framework [18]. In Q-learning technique, an agent studies ideal activities/actions via experimentation communication with its surrounding. On each progress, the agent picks an activity that modifies the condition of the framework via a progress stage, at that point it gets a reward showing how positive or negative the activity was. The agent objective is to strengthen this reward by calculating the ideal approach and picking the best activity for each condition of the framework. The objective of Q-Learning is to gain proficiency with an approach that advises an agent which activity/actions to make under which conditions.

Definition: In Q-learning procedure, an agent attempts to discover the strategy that maximizes the Q-value function which offers the expected utility of choosing an action $a$ in an existing state $s$. 
Formulation: The objective of a Q-learning process is to discover the best strategy $\Pi_{\text {opt }}$ that maximizes the cumulative expected reward (over many trials) in the learning process ( $n$ is the number of trials):

$$
\prod \operatorname{opt}\left(E\left[\sum_{k=0}^{n} \gamma R\left(s_{k}-a_{k}\right)\right]\right)
$$

$\gamma$ which is $(0 \leq \gamma \leq 1)$ represents a discount factor. At learning trial $k$, with an action $a_{k}$ taken in state $s_{k}$, the received reward is represented as $R\left(s_{k}-a_{k}\right)$. For $\gamma=0$ upcoming rewards have no effect on the state value, whereas for $\gamma$ close to 1 , upcoming actions are considered as important as the immediate rewards. A Q-function is defined for a given policy $\Pi$ as:

$$
Q(s, a)=R(s, a)+\gamma \sum_{v \in S} P_{s, v}(a) Q(v, b)
$$

where:

$R(s, a)$ is the expected reward of the current pair of state-action, which represents an action a taken in state environment $\mathrm{s}$

$P_{s, v}(a)$ is the probability of transition from the current state $\mathrm{s}$ to the next state $\mathrm{v}$ as an outcome of action a.

$Q(v, b)$ is the new state-action pairs Q-function value.

To ensure that there is at least one optimal strategy $\Pi^{*}$ in a single agent environment, we apply Bellman's optimality [19]. Q-function maximum value which indicates the optimal action $b$ for every possible next pair $(v, b)$ is denoted as $Q^{*}(s, a)$.

$$
Q^{*}(s, a)=R(s, a)+\gamma \sum_{v \in S} P_{s, v}(a) \max _{b \in A} Q^{*}(v, b)
$$

In an iterative procedure, Q-learning determines the optimal $Q^{*}(s, a)$. At each stage during the learning procedure, the Q-value function should be updated using the (4):

$$
Q_{t}(s, a)=(1-\alpha) Q_{t-1}(s, a)+\alpha\left(R_{t}(s, a)+\gamma \max _{b} Q_{t-1}(v, b)\right)
$$

where $\alpha$ represent the learning rate.

\section{RESEARCH METHOD} as follows:

All parameters related to handover decision phase based on Q-learning technique are defined

a. Environment: involves all components besides the agent

In our framework, it contains the macrocell eNB and all femtocells HeNBs in the UE $\mathrm{eNB}$ 's neighboring cell list (NCL). We consider that the environment is a discrete-time, finite-state and stochastic dynamic system.

b. Agent: is the decision maker

In our case, the agent involves the macrocell mobile user $\mathrm{UE}_{\mathrm{eNB}}$ executing a handover process from its serving cell to another neighboring cell that provide better performance.

c. State: is the environment's current state

In our framework, it involves the current $\mathrm{UE}_{\mathrm{eNB}}$ serving cell, which is the macrocell eNB. The state set $\mathrm{S}$ is defined as $S=\left\{s=1,2, \ldots, N_{N C L}+1\right\}$ where $N_{N C L}$ is the number of neighboring femtocells. $(s=1)$ refers to the initial state where the mobile user UEeNB is connected to the macrocell eNB. To select the target cell in a short time we have to short-list the neighboring femtocells, to optimize the candidate neighboring cell list we propose Distance and moving Direction Q-learning based technique $\left(D^{2} Q\right.$ technique $)$. The UE direction assists the handover decision through avoiding signaling measurement controls with neighbor cells that are not ahead of the UE trajectory as well as in selecting the neighbor cell that fits as the target cell. The distance between UE and target cell is important, which should not exceed the cell radius, in order that cells which are far away from the mobile user are not involved in the candidate neighboring list.

Neighbor cells location and each user equipment UE position are determined using GPS [20]. $\left|\mp \theta_{t h}{ }^{\circ}\right|$ is the range that all nominee cells should be situated ahead of the user equipment UE direction, and each cell that is located inside this zone will have the priority to be combined into the candidate cell list [20]. Assume that a UE is moving from location $\mathrm{P}_{1}$ to location $\mathrm{P}_{2}$ as shown in Figure $2, \mathrm{P}_{3}$ is the neighbor cell location. Every neighbor cell of the user equipment is tested via calculating the angle $\theta$ of $\angle P_{2}, P_{1}, P_{3}$ as following: 


$$
\theta_{p_{2}, p_{1}, p_{3}}=\cos ^{-1} \frac{\left(P_{3}-P_{1}\right) \cdot\left(P_{2}-P_{1}\right)}{\left|P_{3}-P_{1}\right|\left|P_{2}-P_{1}\right|}
$$

where $P_{1}, P_{2}$ and $P_{3}$ are $P_{1}\left(x_{1}, y_{1}\right), P_{2}\left(x_{2}, y_{2}\right)$ and $P_{3}\left(x_{3}, y_{3}\right)$ respectively.

The distance between the user equipment and the neighbor cell is applied, which should not exceed the neighbor cell radius, in order that cells which are far away from the user equipment are not involved in the candidate cell list [21-23]. The distance between the user equipment at position $P_{2}$ and the cell at location $P_{3}$ is calculated by (6):

$$
d_{p_{3}, p_{2}}=\sqrt{\left(x_{3}-x_{2}\right)^{2}+\left(y_{3}-y_{2}\right)^{2}}
$$

For UE moves from position $\mathrm{P}_{2}$ towards neighbor cell located at $\mathrm{P}_{3}$, we consider the neighbour cell to be a candidate cell $\operatorname{if}\left(\theta \leq\left|\mp \theta_{\mathrm{th}}{ }^{\circ}\right|\right)$ and $\left(\mathrm{d}_{\mathrm{p}_{3}, \mathrm{p}_{2}} \leq\right.$ neighbor cell radius $\left.\mathrm{d}_{\mathrm{th}}\right)$. The next stage contains selecting the target cell from the nominee candidate list by utilizing the Weight Adjustment algorithm [20]. In our work, the shortest distance to the user equipment's current position and the narrowest $\theta$ from the candidate cell list would be the most appropriate target cell. The Weight Adjustment algorithm is shown in Algorithm 1.

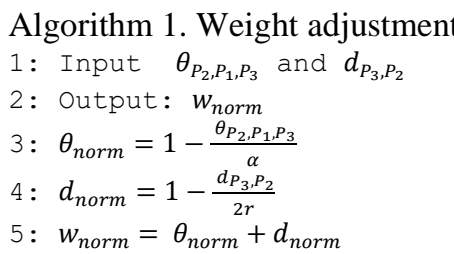

$w_{\text {norm }}$ is used for choosing the target cell. Furthermore, normalization is also implemented for both distance and angle, in order that both will be according to standard integration. For normalization we use $\boldsymbol{\alpha}$ as the angle value. $\theta_{\text {norm }}$ involves the result of angle normalization as all angles of the candidate cells are less than or equal $\left|\mp \theta_{t h}{ }^{\circ}\right|$, this angle $(\boldsymbol{\alpha})$ is used for normalization procedure. $\boldsymbol{d}_{\text {norm }}$ involves the result of distance normalization which is normalized via cell transmission range $(2 \boldsymbol{r})$ to enhance the priority of the angle value, as the distance of all nominee cell list is less or equal to $\boldsymbol{r}$. These methodologies for choosing the candidate cell list and selecting the target cell are illustrated in Algorithm 2.

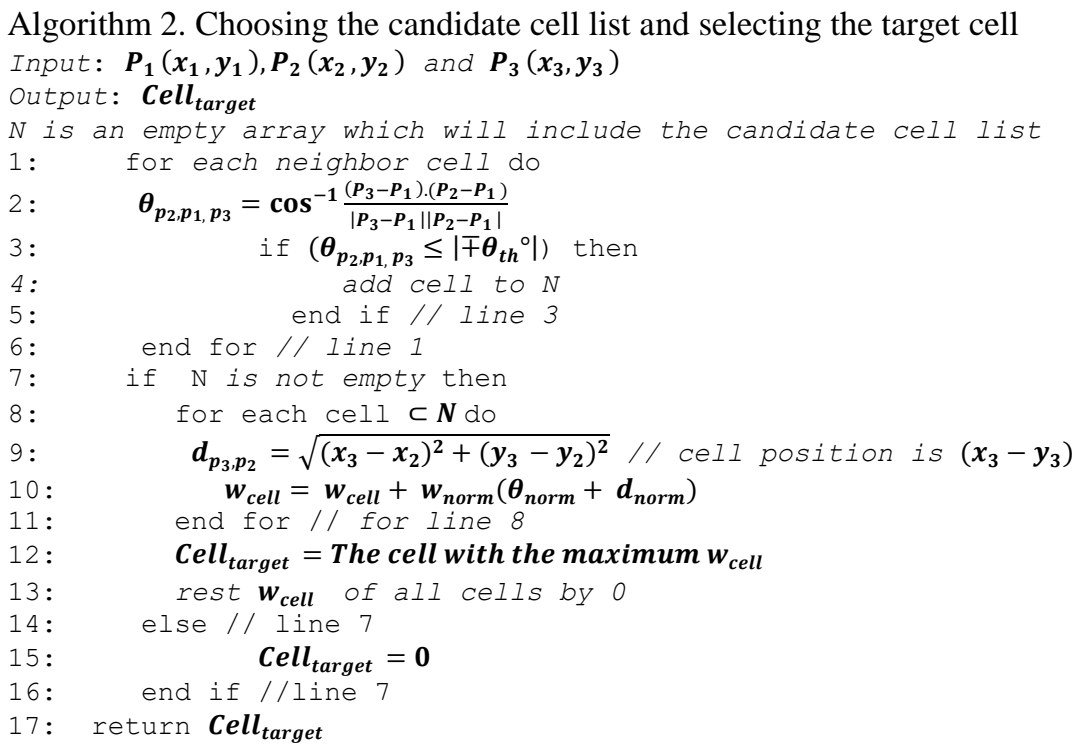

d. Action: is the agent decision result

In our framework, it refers to the handover decision results: the UEeNB may keep its connection with the serving macrocell eNB (action1) or select one of the femtocells HeNBs from its NCL (action $2, \ldots$, action $\mathrm{N}_{\mathrm{NCL}}+1$ ). In our proposal algorithm, we use the $\epsilon$-Greedy technique with an adaptive $\epsilon$ scheme by presenting RSRQ-dependent exploration instead of a fixed or a hand-tuning $\epsilon$ parameter (RSRQ Q-learning based technique (Q2 technique)) [24, 25]. Unlike the traditional $\epsilon$-Greedy method, 
which use a fixed $\epsilon$ parameter, the required action of Q2 technique is to make the agent more explorative in circumstances when the information about the environment is unclear. Q2 technique algorithm is shown in Algorithm 3.

Algorithm 3. $\mathrm{Q}^{2}$ technique

$\Delta \epsilon$ : the amount of decrease or increase of $\epsilon, 0<\epsilon<1$

Stage 1: Set $\Delta \in$ to 0.01 and $\epsilon$ to 0.1

Stage 2: At each trail, we compare $R S R Q_{t-1}$ and $R S R Q_{t}$.

-if $R S R Q_{t-1}<R S R Q_{t}$, then $\epsilon=\epsilon-\Delta \epsilon$

-else $\epsilon=\epsilon+\Delta \epsilon$

e. Reward: It indicates the quality or goodness of the action $a$ in the state $s$, considered as a utility function and denoted by $R$

In our framework, the reward is the earned capacity after connecting to the target cell ( $e N B$ or $H e N B$ ). Our objective is to maintain and maximize the capacity of $U E_{e N B}$ connecting to a new cell after a handover process (Capacity Q-learning based technique (CQ technique)). Thus, if $U E_{e N B}$ selects the macrocell $e N B$ as a serving cell, the utility function $R$ which is a perceived reward (capacity) of the target cell is expressed as 1 . Else if $U E_{e N B}$ selects to connect to one of the femtocells HeNBs in its NCL, the utility function $R$ is expressed as $2[26,27]$.

Let $P_{e N B}$ be the transmitted power by the macrocell eNB and $h_{e N B, k}$ the gain of the channel between the macrocell eNB and its serving $k_{t h}$ macrocell user UE $\mathrm{eNB}_{\mathrm{e}}$. Similarly, $h_{i, j}$ represents the gain of the channel between the $i_{t h}$ femtocell HeNB and the $j_{t h}$ femtocell user $\mathrm{UE}_{H e N B}$. Lastly, $P_{i}$ represents the transmit power of the $i_{t h}$ femtocell HeNB. An Additive White Gaussian Noise (AWGN) is considered at macrocell user $\mathrm{UE}_{e N B}$ with $\sigma^{2}$ power. Macrocell user $\mathrm{UE}_{e N B} k$ capacity from its serving macrocell eNB is calculated by (7):

$$
C_{k}=\frac{B}{N_{U E_{e N B}}} \log _{2}\left(1+\frac{\left|h_{e N B, k}\right|^{2} P_{e N B}}{\sigma^{2}+I}\right)
$$

where $B$ is the available bandwidth, $I=\sum_{i=1}^{N_{H e N B}}\left|h_{i, k}\right|^{2} P_{i}$ is the interference from neighboring femtocells HeNBs, and $N_{H e N B}$ is the number of neighboring femtocells HeNBs. We consider that the bandwidth is equally allocated to all users $\left(\mathrm{UE}_{\mathrm{eNB}}\right.$ and $\left.\mathrm{UE}_{\mathrm{HeNB}}\right)$. The capacity at femtocell user $\mathrm{j}\left(\mathrm{UE}_{\mathrm{HeNB}}\right) j$ from femtocell (HeNB) $i$ is given by (8):

$$
C_{j}=\frac{B}{N_{U E_{H e N B}}} \log _{2}\left(1+\frac{\left|h_{i, j}\right|^{2} P_{i}}{\sigma^{2}+I_{e N B}+I_{H e N B}}\right)
$$

where $I_{e N B}=\left|h_{e N B, j}\right|^{2} P_{e N B}$ is the interference from macrocell eNB, $h_{e N B, j}$ is the gain of the channel between macrocell eNB and user $j$. Also, $I_{H e N B}=\sum_{l \neq i}\left|h_{l, j}\right|^{2} P_{l}$ is the interference from other femtocells HeNBs and $h_{l, j}$ is the gain of the channel between $\mathrm{HeNB}_{l}$, transmitting with power $P_{l}$, and user $j$.

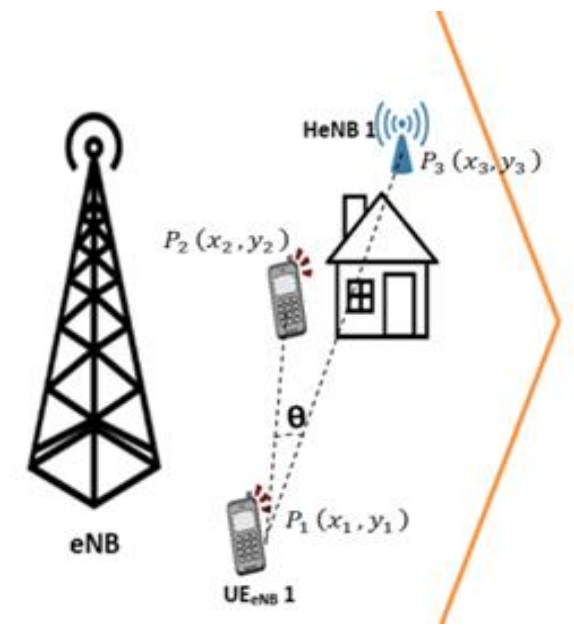

Figure 2. User equipment distance and moving direction 


\section{RESULTS AND DISCUSSION}

The LTE-Sim simulator [28] is used to evaluate the performance of the proposed algorithm depending on the number of the handovers with compare to the algorithm introduced by Suman [17]. The topology consists of two macrocells (eNB) with a radius of $1 \mathrm{~km}$ each and various femtocells (HeNBs) density, the femtocell number is configured as 30, 50, 70 and 90 in each macrocell, and all femtocells are covered by open access type to allow the user equipment UE to handover to each femtocell. Each femtocell radius covers 30 meters. The UE number is configured as 15, 30, 45 and 60. The UEs are distributed randomly in each macrocell coverage area and each UE starts moving from the center of its serving eNB based on random mobility.

The handover decision in the proposed topology will cover three vertical handover types: Hand-in, Hand-between and Hand-out handovers based on the availability of each vertical handover type. Each femtocell will be randomly located between 50 meters to 1000 meters from the macrocell location in three dependent scenarios: close, middle and at the edge. Concerning femtocells distribution scenarios: close, middle and at the edge, femtocells are distributed in four different groups: 30, 50, 70 and 90 in each scenario. Figure 3 presents the average number of handovers for the proposed algorithm in each scenario for 30 UEs. As shown in Figure 3, the relationship between the average number of handovers and femtocells density is positive relationship, which means that the average number of handovers increase when femtocells density increase. While it has the lowest average when the femtocells distribution is at the edge. This is because the mobile users start to move from the location of macrocell tower. In addition, the average of handovers number increases as the number of femtocells in all distribution scenarios increases.

Furthermore, the results of the average number of handovers for the proposed algorithm and Suman handover algorithm were discussed in terms of femtocells that are distributed to groups of 30,50,70 and 90 per each macrocell, and two groups of UEs (15 and 30) as presented in Figure 4. Based on each result, it is evident that by increasing the femtocells number, both algorithms show an increment in the average handovers number, because mobile users make additional handovers with respect to their movements in each mobile user group.

The results emphasize that the best performance was achieved by our algorithm in all distributions of femtocells and all densities. This is because of utilizing Q-learning methodology which allow the mobile user to learn from his previous history, in addition to other supporting methodologies which do not allow the mobile user to connect to femtocells that are only close to the it, but to connect to those located in front of or approximately ahead of current mobile user position in order to avoid the redundant handover.

The user equipment only nominates the femtocell whose tower location is less than $| \pm 25|$ and the distance between the UE and the candidate femtocell is less than or equal 28 meters. On the contrary, in the case of Suman handover decision the handover procedure is triggered when the RSS between the UE and its neighbor femtocells is higher than the RSS between the UE and its serving cell without any consideration of how long the target femtocell will serve the UE and its usefulness to do handover or not.

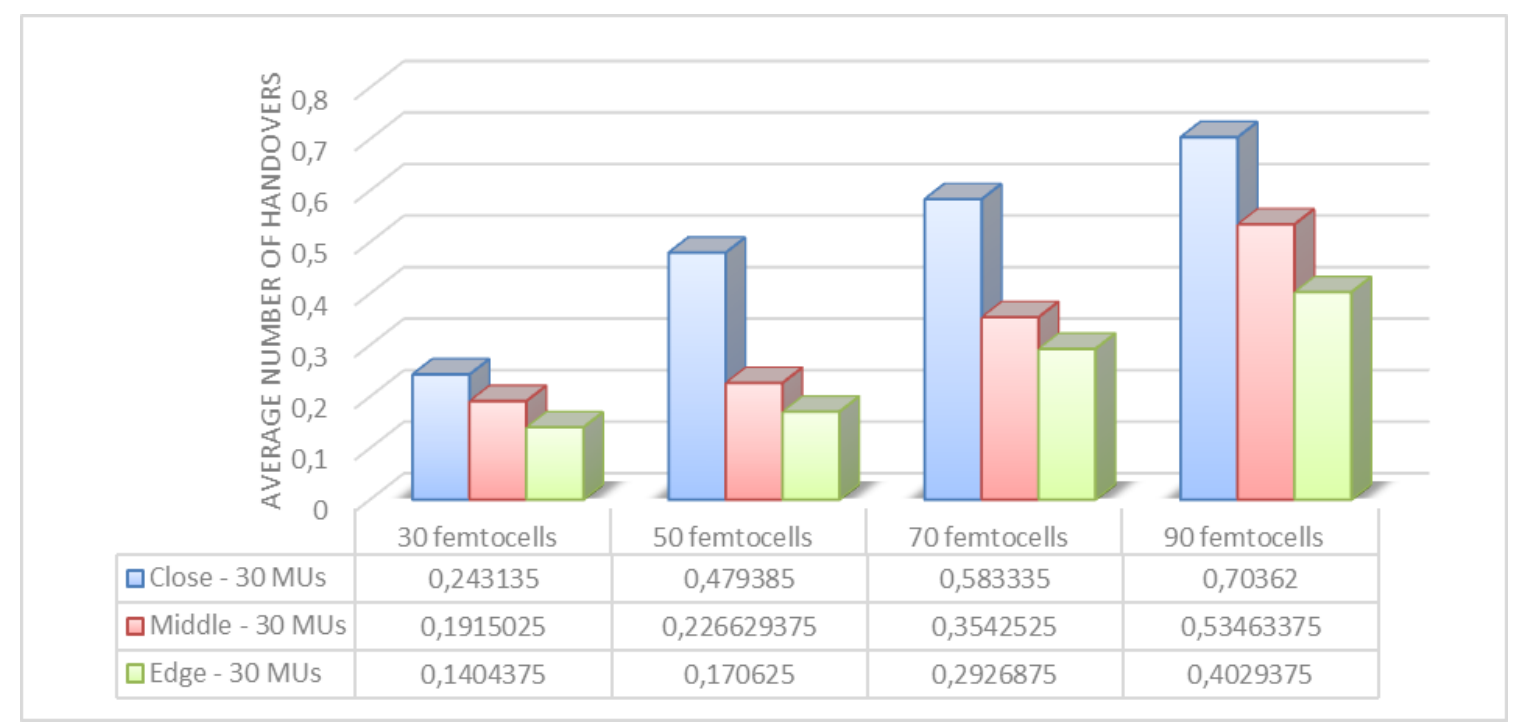

Figure 3. Comparison of average number of handovers of the proposed algorithm in three scenarios: close, middle and at the edge 


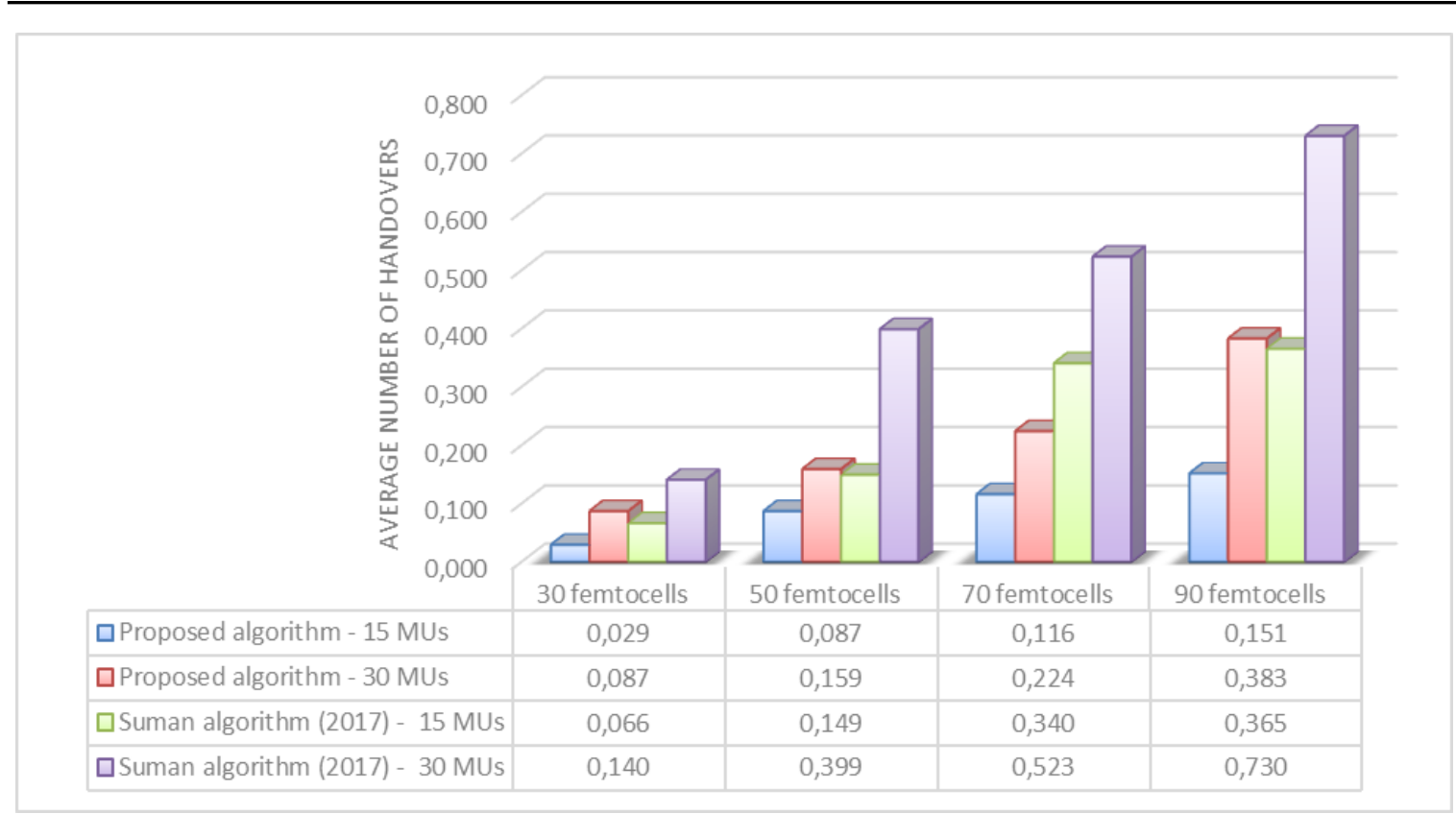

Figure 4. Comparison of average number of handovers for both algorithms

Finally, regarding the total average number of handovers for each UE group, it is reduced in the proposed algorithm by (55.63\%) compared to Suman handover algorithm for all various femtocells densities when the number of UE is 15 . Moreover, the proposed algorithm reduces the total average number of handovers by (41.74\%) compared to Suman handover algorithm for all various femtocells densities when the UEs number is 30 .

\section{CONCLUSION AND FUTURE WORK}

The simulation results show that the proposed algorithm performs well in enhancing the handover decision in LTE-A networks. The simulation results examined the proposed algorithm for femtocells of the open access type in order to enhance the target femtocell selection in the vertical handover decision. The selection of suitable parameters to improve the handover decision still encompasses a wide area research. Therefore, the recommendation for further research in this field can be as follows: Firstly, is to investigate different parameters of user performance in light of handover and load balancing in the wireless system over horizontal and vertical networks. Secondly, to investigate different parameters of user performance on both femtocell types: the close and hybrid. Finally, in regard to implementation, UE velocity should be taken into account in the handover decision as the main behavior. Thus, by monitoring the three main behaviors at UE which are the UE mobility, acceleration, and deceleration as the frequent line changes, the suitability of the proposed algorithm for the UE behavior can be ensured.

\section{ACKNOWLEDGEMENTS}

The authors would like to express the gratitude to the Ministry of Education, Malaysia and Universiti Teknologi MARA, Selangor, Malaysia for the financial support given for this project (Geran Bestari Perdana) [File No: 600-IRMI/PERDANA 5/3 BESTARI (095/2018).

\section{REFERENCES}

[1] B. Ma, et al., "Modeling and Analysis for Vertical Handoff Based on the Decision Tree in a Heterogeneous Vehicle Network," IEEE Access, vol. 5, pp. 8812-8824, 2017.

[2] T. Zahir, et al., "Interference Management in Femtocells," IEEE Communications Surveys \& Tutorials, vol. 15, no. 1, pp. 293-311, 2013.

[3] G. Godor, et al., "A Survey of Handover Management in Lte-Based Multi-Tier Femtocell Networks: Requirements, Challenges and Solutions," Computer Networks, vol. 76, pp. 17-41, 2015. 
[4] S. Bhosale and R. Daruwala., "Multi-criteria Vertical Handoff Decision Algorithm Using Hierarchy Modeling and Additive Weighting in an Integrated WLAN/WiMAX/UMTS Environment-A Case Study," KSII Transactions on Internet \& Information Systems, vol. 8, no. 1, pp. 35-57, 2014.

[5] N. Rajule, et al., "Survey of vertical handover decision algorithms," International Journal of Innovations in Engineering and Technology (IJIET), vol. 2, no. 1, pp. 362-368, 2013.

[6] H. Kwak, et al., "Mobility management survey for home-eNB based 3GPP LTE systems," Journal of Information Processing Systems, vol. 4, no. 4, pp. 145-152, 2008.

[7] S. Neeraja, et al., "Analysis of Adaptive Hysteresis Based Horizontal Handoff Algorithm for GSM," International Journal of Innovative Research in Electrical, Electronics, Instrumentation and Control Engineering, vol. 1, no. 9, pp. 433-437, 2013.

[8] X. Yan, et al., "A survey of vertical handover decision algorithms in fourth generation heterogeneous wireless networks," Computer Networks, vol. 54, no. 11, pp. 1848-1863, 2010.

[9] A. Gharsallah, et al., "Network Selection in Heterogeneous Wireless System Environments," Journal of Networks, vol. 10, no. 12, pp. 633-641, 2015.

[10] S. Ciochina, et al., "An Optimized Nlms Algorithm for System Identification," Signal Processing, vol. 118, pp. 115-121, 2016.

[11] Y. C. Wang and C. A. Chuang, "Efficient Enb Deployment Strategy for Heterogeneous Cells in 4G LTE Systems," Computer Networks, vol. 79, pp. 297-312, 2015.

[12] M. H. Hachemi, et al., "Predicting the Probability of Spectrum Sensing with Lms Process in Heterogeneous LTE Networks," Radioengineering, vol. 25, no. 4, pp. 808-820, 2016.

[13] H. Ge, et al., "A history-based handover prediction for LTE systems," 2009 International Symposium on Computer Network and Multimedia Technology, pp. 1-4, 2009.

[14] Y. H. Wang, et al., "A handover prediction mechanism based on LTE-A UE history information," 2014 International Conference on Computer, Information and Telecommunication Systems (CITS), pp. 1-5, 2014.

[15] X. Chen, et al., "Efficient and prompt handover in LTE-based systems by predicting the target eNodeBs," 2014 International Conference on Cyber-Enabled Distributed Computing and Knowledge Discovery, pp. 406-413, 2014.

[16] F. M. Chang, et al., "An efficient handover mechanism by adopting direction prediction and adaptive time-totrigger in LTE networks," International Conference on Computational Science and Its Applications, vol. 7975, pp. 270-280, 2013.

[17] S. Deswal and A. Singhrova, "A Vertical Handover Algorithm in Integrated Macrocell Femtocell Networks," International Journal of Electrical and Computer Engineering (IJECE), vol. 7, no. 1, pp. 299-308, 2017.

[18] E. Alpaydin, "Introduction to machine learning," MIT press, 2nd edition, 2010.

[19] C. J. C. H. Watkins and P. Dayan, "Technical note: Q-learning," Machine Learning, vol. 8, pp. 279-292, 1992.

[20] Y. S. Huang, et al., "A Handover Scheme for LTE Wireless Networks under the Assistance of GPS," Proceeding 2013 8th International Conference on Broadband and Wireless Computing, Communication and Applications, BWCCA, pp. 399-403, 2013.

[21] A. Bogdanov, "Location identification and handover in new-generation mobile networks," 2020 Moscow Workshop on Electronic and Networking Technologies (MWENT), Moscow, Russia, pp. 1-4, 2020

[22] M. A. Wong, J. A. J Alsayaydeh, S. M. Idrus, N. Zulkifli, and M. Elshaikh, "Efficient P2P data dissemination in integrated optical and wireless networks with Taguchi method," TELKOMNIKA Telecommunication, Computing, Electronics and Control, vol. 17, no. 4, pp. 1642-1647, 2019.

[23] K. Ahuja, et al., "Network Selection Based on Weight Estimation of QoS Parameters in Heterogeneous Wireless Multimedia Networks," International Journal of Wireless Personal Communications, vol. 77, no. 4, pp. 3027-3040, 2014.

[24] H. A. Mahmoud, et al., "Performance of Open Access Femtocell Networks with Different Cell-Selection Methods," in 2010 IEEE Vehicular Technology Conference, pp. 1-5, 2010.

[25] C. Dhahri and T. Ohtsuki, "Learning-based Cell Selection Method for Femtocell Networks," IEEE Vehicular Technology Conference, pp. 1-5, 2012.

[26] B. Liu, et al., "AHP and Game Theory based Approach for Network Selection in Heterogeneous Wireless Networks," IEEE Consumer Communications and Networking Conference (CCNC), Las Vegas, Nevada, USA, pp. 501-506, 2014.

[27] V. A. Narayanan, et al., "An Intelligent Vertical Handover Decision Algorithm for Wireless Heterogeneous Networks," American Journal of Applied Sciences, vol. 11, no. 5, pp. 732-739, 2014.

[28] G. Piro, et al., "Simulating LTE cellular systems: an open source framework," IEEE Transactions on Vehicular Technology, vol. 60, no. 2, pp. 498-513, 2011. 